Relations industrielles

Industrial Relations

\title{
Programmes d'emplois compensatoires, par E. Jay Howenstine, O.C.D.E., Paris, 1967, 52 pages.
}

\section{Pierre Dionne}

Volume 23, numéro 3, 1968

URI : https://id.erudit.org/iderudit/027938ar

DOI : https://doi.org/10.7202/027938ar

Aller au sommaire du numéro

Éditeur(s)

Département des relations industrielles de l'Université Laval

ISSN

0034-379X (imprimé)

1703-8138 (numérique)

Découvrir la revue

Citer ce compte rendu

Dionne, P. (1968). Compte rendu de [Programmes d'emplois compensatoires, par E. Jay Howenstine, O.C.D.E., Paris, 1967, 52 pages.] Relations industrielles /

Industrial Relations, 23(3), 520-521. https://doi.org/10.7202/027938ar

Tous droits réservés @ C Département des relations industrielles de l'Université Laval, 1968
Ce document est protégé par la loi sur le droit d'auteur. L’utilisation des services d'Érudit (y compris la reproduction) est assujettie à sa politique d'utilisation que vous pouvez consulter en ligne.

https://apropos.erudit.org/fr/usagers/politique-dutilisation/ 
soi. Quatre autres hypothèses postulant des relations entre les variables énoncées plus hout sont aussi confirmées par les données recueillies.

A la fin de leur étude, les auteurs en profitent pour améliorer leur modèle initial en distinguant entre récompenses intrinsèques et extrinsèques.

D'autres précisions viennent s'ajouter qui ouvrent de nouvelles avenues pour la recherche. Enfin, le dernier chapitre traite des implications de la recherche et présente une courte liste de recommandations qui invitent les dirigeants à questionner le bienfondé de leurs politiques de personnel.

Non seulement pour le praticien des relations industrielles, mais aussi pour ceux qui s'intéressent au développement des sciences du comportement, la lecture de ce livre qui, ò certains endroits, n'est pas d'accès facile il faut l'avouer, provoquera un éveil, puisque plusieurs conclusions viennent bousculer les résultats d'études antérieures qui constituaient l'évangile de l'enseignement et de la pratique.

\section{Lourent BELANGER}

\section{Adaptation des travailleurs au progrès tech- nique au niveau de l'entreprise, Séminaires internationaux 1966-5, Rapport final, O.C.D.E., Paris, 1967, 132 pages.}

Quelle est la réaction du travailleur devant I'innovation technique? Comment l'encourager d̀ y participer au lieu d'y résister? Comment le législateur peut-il minimiser le coût social de l'opération? En d'autres termes, quels sont les principes, les méthodes ot les techniques de planification susceptibles d'apporter I'harmonie et la coordination là où un conflit semble inévitable? L'adaptation de l'homme à l'innovation, tel est l'aspect sur lequel est concentrée cette étude.

La première partie de cet ouvrage a trait à l'attitude des travailleurs et des employeurs à l'égard du progrès technique. L'attitude des travailleurs o le plus, cependant, retenu l'attention.

La partie II étudie les moyens de coordonner l'évolution technique et l'adaptation de la main-d'oeuvre à l'aide de monographies diverses portant sur l'Europe occidentale et, dans un cas, sur le Japon.

La troisième partie traite des techniques d'adaptation individuelle que suppose le pro- grès. Il ne s'agit pas seulement de l'adaptation au niveau de l'entreprise (ré-affectation, reclassement, retraite anticipée, etc.), mais aussi, au niveau de la branche d'activité et de l'économie, de l'adaptation de la structure des salaires, de la formation industrielle, et des régimes d'indemnités de licenciement.

Le présent rapport étudie le problème essentiellement au niveau de l'entreprise, mais il ne faut pas s'en tenir strictement à ce point de vue. L'action de chaque entreprise a des répercussions, non seulement sur son personnel, mais oussi sur les outres entreprises et parfois sur l'économie toute entière. D'autre part, l'action des pouvoirs publics a une incidence sur les entreprises, quelle que soit leur dimension. Pour tirer le parti maximum des programmes d'adaptation des entreprises et de ceux des pouvoirs publics, il faut évidemment qu'ils soient coordonnés. Tel est le thème de la partie IV.

C'est au niveau de la coordination des programmes des entreprises et de ceux des pouvoirs publics qu'on peut vraiment parler de politiques de la main-d'oeuvre. Celles-ci doivent, avant tout, tendre au développement et à l'emploi optimal des ressources humaines, tant pour accélérer la croissance économique que pour élever les riveaux de vie. L'automation est un moyen d'y arriver. Mais la conclusion qui se dégage de cette étude est qu'en l'absence de bonnes politiques de l'emploi et de la main-d'oeuvre, le processus indispensable d'adaptation à l'automation sera laborieux et difficile, tant pour les travailleurs que pour les employeurs, et qu'il imposera une lourde charge à la collectivité.

Cet ouvrage s'adresse particulièrement, ò notre avis, à tous les dirigeants d'entreprise aux prises avec de tels problèmes.

\section{Pierre DIONNE}

Programmes d'emplois compensatoires, par E. Jay Howenstine, O.C.D.E., Paris, 1967, 52 pages.

Cette petite plaquette est une version abrégée du rapport qui a paru sous le même titre en tant que No 3 de la série Développement des possibilités d'emploi. Les deux premières publications s'intitulaient: « Aménagement économique et social du territoire et "Méthodes de sélection des industries pour les régions en stagnation $»$. Après en avoir fait un bref historique, l'auteur pose 
le problème de la définition et du rôle des programmes d'emplois compensatoires à partir des diverses conceptions du sujet. Dans un deuxième temps, l'auteur traite d'abord des caractéristiques des ressources nécessaires ò de tels programmes et des contraintes techniques existantes. II passe ensuite à la mise en oeuvre des programmes d'emplois compensatoires et souligne les contraintes institutionnelles contre lesquelles it fout lutter. L'auteur termine son étude en discutant de la place de ces programmes dans une politique économique nationale et de leurs incidences sur les autres objectifs nationoux.

Cette version abrégée donne une excellente vue d'ensemble du problème, mais demande, par contre, d'être lue avec beaucoup d'attention.

\section{Pierre DIONNE}

Conseil pour les groupes spéciaux, par Gertrude Williams, O.C.D.E., Paris, 1967, 127 pages.

La présente étude est destinée à déterminer s'il est possible d'accroître la productivité de la main-d'oeuvre en réduisant le chômage à long terme et en dissuadant les travailleurs de changer trop fréquemment d'emplois, au moyen de l'organisation des conseillers spéciaux. En effet, l'objet de l'étude est d'examiner le rôle que peut jouer le service de l'emploi pour aider les chômeurs à long terme et les travailleurs changeant fréquemment d'emploi. D'autre part, I'ouvrage est basé sur l'hypothèse que le Service de l'emploi pourrait être chargé de dépister, d'interviewer et de conseiller les personnes depuis longtemps en chômage puisqu'il est le point de rencontre des travailleurs en quête d'emploi et de ceux qui ont été invités ò s'adresser à lui par d'autres organismes. L'auteur traite tour à tour du réemploi des cas difficiles, du rôle des conseils pour le placement des cas spéciaux, du service de l'emploi versus les servires sociaux, de la consultation, et du contrôle des résultats avant d'affirmer, en conclusion, la nécessité des services spéciaux de consultation. Tous les responsables des Services de l'emploi (ou Bureau de placement) auraient avantage à lire cette étude.

\section{Pierre DIONNE}

Combien vaut notre entreprise?, par André Barnay et Georges Calba, Entreprise Moderne d'Edition, Poris, 1968.

$\mathrm{Ce}$ livre se veut une revue des diverses méthodes d'évaluation des experts et de leurs principes sous-jacents. Ajoutons de suite que l'expert lui-même n'apprendra à peu près rien de neuf, sinon la simplicité. Cet ouvrage, en effet, s'adresse aux noninitiés, aux non-spécialistes qui s'affolent devant la kyrielle des solutions proposées. André Barnay et Georges Calba écrivent: "Plus praticiens que théoriciens, notre ambition n'a pas été de faire oeuvre savante mais de jeter un peu de clarté sur ce sujet difficile $\gg$.

La première section s'attarde à la notion générale de l'évaluation. Quelle en est le but? Quelles en sont les valeurs? En quoi consistent les principales phases de l'expertise? II faudra d'abord chercher à connaître l'entreprise elle-même: sa rentabilité, sa structure, son bilan, sa politique financière, ses besoins. La deuxième partie de ce livre s'avèrera peut-être la plus utile pour nos chefs d'entreprise: il s'agit de la pratique de l'évaluation. Comment déterminer l'actif net réel, la valeur substantielle, le rendement financier? Autant de questions, autant de réponses claires et précises. Avec ces nombreux tableaux et ces nombreux exemples chiffrés, cet ouvrage apportera sans aucun doute plus de lumière sur les principes et les méthodes d'expertise les plus couramment utilisés.

\section{Paul GUY}

La conciliation des conflits collectifs du travail en Belgique, par Eliane Vogel-Polsky avec le concours du Centre national de sociologie du droit social, Editions J. Duculot - S.A. Gembloux, Bruxelles, 1966,215 pages.

Tout au long de cet ourrage, l'auteur souligne la complexité des institutions, des techniques et des solutions destinées à résoudre pacifiquement les conflits collectifs du travail.

La première partie de l'étude procède à l'analyse des méthodes les plus usuelles de règlement pacifique des conflits collectifs du travail. L'auteur souligne l'importance du contexte socio-économique pour l'étude des systèmes de prévention des conflits et relève la dualité des procédures officielles et volontaires. Après avoir parcouru les étapes historiques de la solution pacifique des con- 